\title{
From the Clinic
}

\section{Gross hematuria after kidney transplantation}

A 42-year-old male with a history of end-stage kidney disease secondary to diabetes mellitus type 1 received a deceased donor simultaneous kidney/pancreas transplant 10 months prior to presentation. The patient had prompt graft function and no other complications. He presented with gross hematuria and kidney allograft dysfunction with serum creatinine elevation from baseline of 114.92 to $221 \mu \mathrm{mol} / \mathrm{L}$ ( 1.3 to $2.5 \mathrm{mg} / \mathrm{dL}$ ). Ultrasound and abdominal computed tomography were normal. Polyomavirus and cytomegalovirus (CMV) polymerase chain reaction (PCR) were negative. A kidney biopsy showed patchy necrotizing and vaguely granulomatous tubulointerstitial inflammation (Figure 1A). The infiltrate consisted of neutrophils, macrophages and lymphocytes with abundant epithelial cell destruction. Occasional epithelial cell nuclei had glassy chromatin suggestive of viral cytopathic effect (Figure 1B). Immunohistochemical staining for C4d, SV40, CMV and HSV was negative, while staining for adenovirus showed strong reactivity. Positivity of adenovirus PCR of
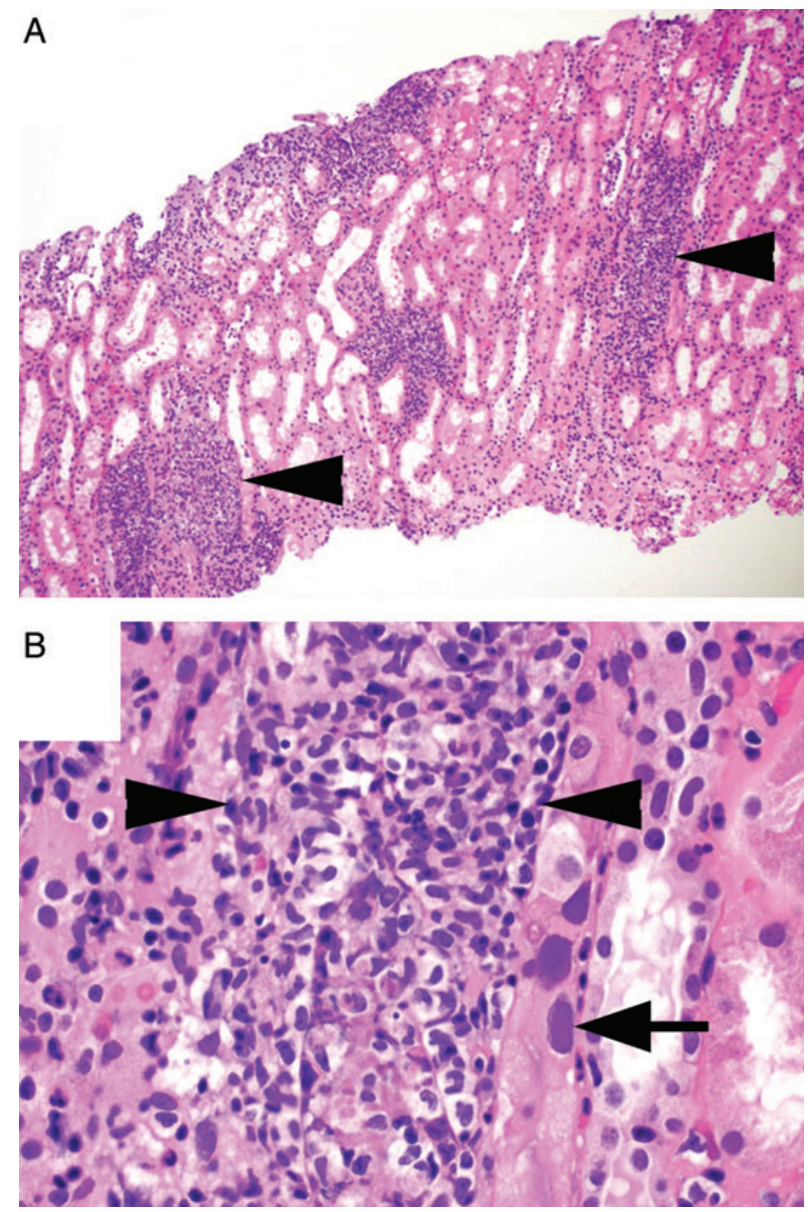

Fig. 1. (A). Lower power image showing patchy tubulointerstitial inflammation (H\&E, $\times 40)$. (B). Tubular epithelial cell necrosis (arrow heads), and a glassy viral inclusion (arrow) (H\&E, $\times 400)$. more than 1000000 copy/mL confirmed the diagnosis of adenovirus tubulointerstitial nephritis. Immunosuppressive medications were reduced and one dose of cidofovir and intravenous immunoglobulin (IVIG) was given. Adenovirus PCR decreased to 175000 after 1 week, and was no longer detected after 1 month. Creatinine gradually decreased to baseline over 3 months.

Adenoviruses are non-enveloped double-stranded DNA viruses. Such virus presents as a self-limited respiratory, gastrointestinal and conjunctival disease in immunocompetent patients. In kidney transplant recipients, it manifests as allograft dysfunction, and hemorrhagic cystitis, but can result in severe fatal cases [1]. Infection is rare with an incidence rate of around $4 \%$ in kidney transplant recipients [2]. Infection can be de novo, or through reactivation of a latent infection of the recipient or from the allograft. Adenovirus serology is not routinely checked before transplantation, but serology mismatch might be a risk factor for infection [1]. The presence of patchy necrotizing infection, large nuclei with basophilic glassy inclusions and negativity of polyoma and CMV serology should suggest additional work-up for adenovirus, especially in the setting of hemorrhagic cystitis. A PCR offers a highly sensitive tool to confirm the infection and monitor the response of treatment [3]. Management starts with reduction of immunosuppressive medications. Severe cases might require one or two regimens of cidofovir, and administration of IVIG. Outcomes of reported cases showed that clearance of adenovirus viremia occurs within 1-3 weeks [2], and that there is an improvement in kidney allograft function to baseline or near baseline in the majority of cases [4].

Conflict of interest statement. None declared.

${ }^{1}$ Division of Nephrology, Mayo Clinic, Scottsdale, Arizona, USA

Tarek Alhamad ${ }^{1}$

${ }^{2}$ Department of Laboratory Medicine Maxwell L. Smith ${ }^{*}$ and Pathology, Mayo Clinic,

Scottsdale, Arizona, USA

"Correspondence and offprint requests to: Maxwell L. Smith.

E-mail: smith.maxwell@mayo.edu

\section{References}

1. Echavarria M. Adenoviruses in immunocompromised hosts. Clinical microbiology reviews. 2008; 21: 704-715 Epub 2008/10/ 16

2. Watcharananan S, Avery R, Ingsathit $A$ et al. Adenovirus disease after kidney transplantation: course of infection and outcome in relation to blood viral load and immune recovery. Am J Transplant 2011; 11: 1308-1314

3. Florescu MC, Miles CD, Florescu DF. What do we know about adenovirus in renal transplantation? Nephrol Dial Transplant 2013; 28: 2003-210 Epub 2013/03/16

4. Hofland CA, Eron LJ, Washecka RM. Hemorrhagic adenovirus cystitis after renal transplantation. Transplant Proc 2004; 36: 3025-3027 Epub 2005/02/03

doi: $10.1093 / \mathrm{ckj} / \mathrm{sft} 127$

Advance Access publication 7 November 2013 